\title{
The Superstable 25 kDa Monolayer Protected Silver Nanoparticle: Measurements and Interpretation as an Icosahedral $\mathrm{Ag}_{152}\left(\mathrm{SCH}_{2} \mathrm{CH}_{2} \mathrm{Ph}\right)_{60}$ Cluster
}

\author{
Indranath Chakraborty, ${ }^{\dagger, \|}$ Anuradha Govindarajan, ${ }^{\dagger, \|, \perp}$ Jayanthi Erusappan, ${ }^{\dagger, \perp}$ Atanu Ghosh, ${ }^{\dagger}$
} T. Pradeep, ${ }^{* \dagger}$ Bokwon Yoon, ${ }^{\ddagger}$ Robert L. Whetten, ${ }^{\S}$ and Uzi Landman ${ }^{\ddagger}$

${ }^{\dagger}$ DST Unit of Nanoscience (DST UNS), Department of Chemistry, Indian Institute of Technology Madras, Chennai 600036, India

${ }^{\ddagger}$ School of Physics, Georgia Institute of Technology, Atlanta, Georgia 30332-0430, United States

${ }^{\S}$ School of Chemistry \& Biochemistry, Georgia Institute of Technology, Atlanta, Georgia 30332, United States

Supporting Information

ABSTRACT: A cluster obtained in high yield from the reduction of a silver-thiolate precursor, $\mathrm{Ag}-\mathrm{SCH}_{2} \mathrm{CH}_{2} \mathrm{Ph}$, exhibited a single sharp peak near $25 \mathrm{kDa}$ in the matrix-assisted laser desorption mass spectrum (MALDI MS) and a welldefined metal core of $\sim 2 \mathrm{~nm}$ measured with transmission electron microscopy (TEM). The cluster yields a single fraction in high-performance liquid chromatography (HPLC). Increased laser fluence fragments the cluster until a new peak near $19 \mathrm{kDa}$ predominates, suggesting that the parent cluster$\mathrm{Ag}_{152}\left(\mathrm{SCH}_{2} \mathrm{CH}_{2} \mathrm{Ph}\right)_{60}$ - evolves into a stable inorganic core$\mathrm{Ag}_{152} \mathrm{~S}_{60}$. Exploiting combined insights from investigations of clusters and surface science, a core-shell structure model was developed, with a 92-atom silver core having icosahedral-

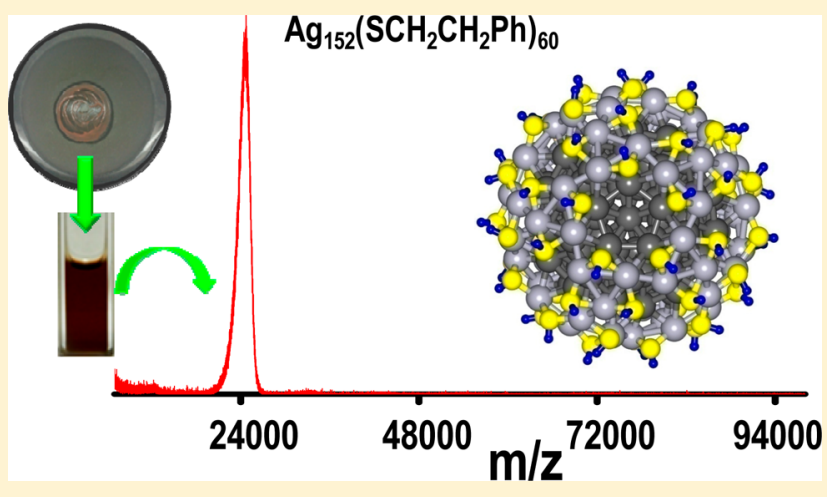
dodecahedral symmetry and an encapsulating protective shell containing $60 \mathrm{Ag}$ atoms and 60 thiolates arranged in a network of six-membered rings resembling the geometry found in self-assembled monolayers on $\operatorname{Ag}(111)$. The structure is in agreement with small-angle X-ray scattering (SAXS) data. The protective layer encapsulating this silver cluster may be the smallest known three-dimensional self-assembled monolayer. First-principles electronic structure calculations show, for the geometry-optimized structure, the development of a $\sim 0.4 \mathrm{eV}$ energy gap between the highest-occupied and lowest-unoccupied states, originating from a superatom 90-electron shell-closure and conferring stability to the cluster. The optical absorption spectrum of the cluster resembles that of plasmonic silver nanoparticles with a broad single feature peaking at $460 \mathrm{~nm}$, but the luminescence spectrum shows two maxima with one attributed to the ligated shell and the other to the core.

KEYWORDS: Noble metals, clusters, nanoparticles, plasmon resonance, MALDI MS, first-principles electronic structure, projected electronic density of states (PDOS), superatom electronic shells

$\mathrm{T}$ he quest to fabricate, characterize, and understand small noble metal molecules is among the outstanding challenges of contemporary materials science, as such nanomaterials bridge the atomic and the bulk worlds. ${ }^{1-3}$ Distinct stable clusters most often arrange in concentric atomic shell structures composed of $13,55,147,309$, and so forth, atoms, known as Mackay icosahedra, and some of them have been synthesized through solution chemistry. Whereas reports about $\mathrm{Au}_{13}{ }^{4}$ and $\mathrm{Au}_{55}{ }_{5,6}$ are available, investigations of the corresponding silver clusters remain an open challenge. Recently, several studies pertaining to electronically stable clusters of gold and silver have appeared. ${ }^{7-16}$ In some of these investigations (particularly on gold clusters, starting with an early study of bare gold clusters), ${ }^{17}$ a superatom description of the enhanced stability acquired via electron shell closures at magic number sizes, that is, for clusters containing 2, 8, 18, 34, 58,92 , and so forth, delocalized electrons (see below), has been applied with a significant measure of success; we recall here that this methodology has been formulated in the mid 1980s to explain time-of-flight mass spectrometric measurements on bare alkali-metal clusters. ${ }^{18}$ The scarcity of reports on silver clusters $^{14-16,19,20}$ may be attributed to the high reactivity and relatively poor stability of the Ag-ligand interface. Consequently, the creation, characterization, and understanding of stable silver clusters would provide a "missing link" in this field and serve as impetus for increased research activity in this area.

In this Letter, we present the successful isolation and identification of a $25 \mathrm{kDa}$ silver-thiolate cluster, 1, which points to the existence of an $\mathrm{Ag}$ analogue of the familiar

Received: August 29, 2012

Revised: October 15, 2012 
$\mathrm{Au}_{144 / 146}$ (thiolate) 60 systems. ${ }^{21-24}$ Optical absorption of the cluster closely resembles the plasmon resonance of silver nanoparticles and confirms that molecular excitations in such systems resemble free electron oscillations in metals.

The synthesis of $\mathbf{1}$ follows a solid state route ${ }^{14}$ in which $\mathrm{AgNO}_{3}(\mathrm{~s})$ was ground with phenylethanethiol (PETH)(l) at 1:5.3 molar ratio in a mortar and pestle. Adding $25 \mathrm{mg}$ of $\mathrm{NaBH}_{4}(\mathrm{~s})$ and continued grinding completes the reaction. Immediate extraction of the excess thiol in the reaction mixture with $5 \mathrm{~mL}$ of ethanol and subsequent dissolution of the residue in toluene/tetrahydrofuran (THF) makes product $\mathbf{1}$ [details are in SI (S1)]. The crucial aspect of this procedure is the limited supply of water needed for the reduction, which becomes available from the laboratory atmosphere as well as from the ethanol used for subsequent washing. Extended grinding in humid air as well as repeated washing by ethanol stimulate cluster formation and subsequently nanoparticles were detected. The sample was further purified using HPLC (see Figure S2), showing a single sharp peak consistent with the formation and isolation of a unique compound.

Mass spectrometry is the key analytical technique used to obtain the molecular composition of clusters of this kind. ${ }^{23,25,26}$ In this case, laser desorption at threshold laser power (fluence) gives molecular ion features in MALDI MS (Figure 1), although at higher fluence dissociation is seen. The spectra shown in Figure 1 are at the lowest fluence needed to observe ion signals (data at higher fluence will be discussed below). We show here mass spectra for the unpurified (Figure 1a) as well as the HPLC-purified sample (Figure 1b). The peak in Figure 1a is centered at $m / z=24600 \mathrm{Da}$ (fwhm of $1.5 \mathrm{kDa}$ ), where the imprecision in the mass number is \pm 100 in this mass range, and therefore the composition will have corresponding uncertainty. Additionally, the doubly charged cluster $\left(2^{+}\right.$, dication $)$is observed (see small signal peaked at $\mathrm{m} / z 12300 \pm 30$ ). The negative ion mass spectra were of much lower intensity, but the features were identical. The peak of the data in Figure $1 \mathrm{~b}$ is located at $m / z=24610 \pm 80$, and it is narrower (fwhm of 1.3 $\mathrm{kDa}$ ), exhibiting in addition a much more pronounced dication signal with a peak at $12320 \pm 30$. Such a sharp and solitary peak in the mass spectrum has not been observed for any other large silver cluster to date. Moreover, the above observation of a clear dication signal corraborates the unit charge assignment of the cluster molecular ion. The similarity between the results with and without HPLC purification allows us to focus in the following on measurements for the latter.

The estimated yield of the reaction was determined to be as high as $82 \%$. These factors suggest the ubiquitous existence of an uniquely stable cluster compound lying in this particular size and composition range. A well-defined cluster was also supported by the HRTEM image (Figure 1c), which shows the presence of a core with an average diameter of $\sim 2 \mathrm{~nm}$. SAXS (small angle X-ray scattering) analysis in solution further confirms the homogeneity of particle size with a sharp feature at a radius of $1.395 \mathrm{~nm}$ (Figure S3). Some bigger particles of 3-4 $\mathrm{nm}$ diameter were observed in TEM (Figure 1) which are attributed to cluster aggregates or cluster coalescence which is typical in the electron microscopic analysis of clusters, especially with higher primary beam energy. Interestingly, this cluster shows a specific arrangement in HRTEM, which indicates a high propensity for crystallization. This specific arrangement was not restricted to a single place but was found almost throughout the entire grid (Figure 1c). Even the SEM images showed some crystalline behavior along with sharp faces
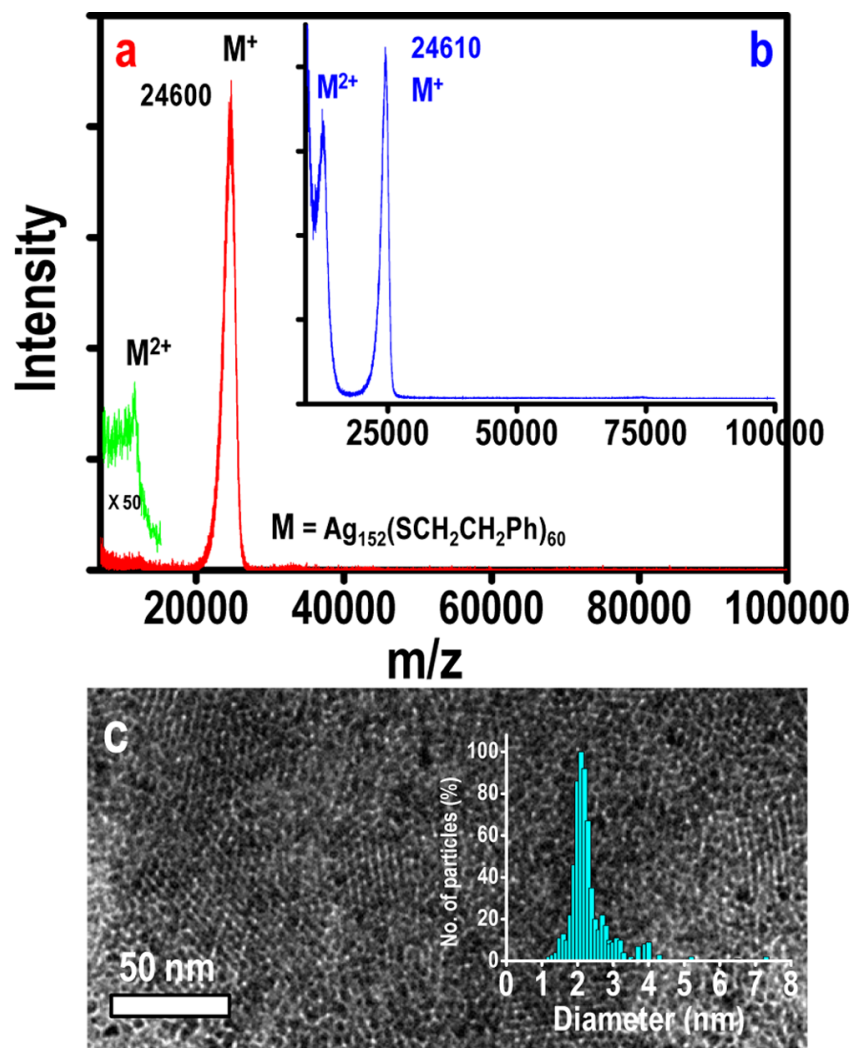

Figure 1. (a) MALDI MS mass spectrum of as-synthesized $25 \mathrm{kDa}$ compound 1 (extracted in toluene) detected in the positive mode. It gives a sharp (fwhm $m / z 1.5 \mathrm{kDa}$ ) molecular peak centered at $\mathrm{m} / z=$ $24600 \pm 100$. A minor peak at $m / z 12300 \pm 30$ was seen, due to the doubly charged species. For clarity, this was expanded 50 times in the vertical axis. (b) Same as a but for a HPLC-purified sample extracted in THF, showing a narrower peak (fwhm of $1.3 \mathrm{kDa}$ ) at $24610 \pm 80$ $\mathrm{Da}$ and a pronounced dication peak at $m / z=12320 \pm 30$ mass. (c) HRTEM image of clusters that are drop-cast on a carbon-coated copper grid. This specific arrangement of clusters has been observed throughout the grid. Inset shows the size distribution of the clusters observed in TEM. A very narrow distribution was found with an average core diameter of $\sim 2 \mathrm{~nm}$.

(Figure S4) of microcrystals. Elemental analysis (Figure S5) of these crystallites showed the expected quantity of silver and sulfur.

Systematic studies of the dependence on laser fluence $(f)$ were performed to establish the character of the molecular ion feature in the MALDI MS. Below a well-defined threshold laser fluence, $f_{\mathrm{TH}}$, no ions were detected (Figure S6), and above a second threshold fragmentation commenced (Figure S7). With further increased fluence, $f=x f_{\mathrm{TH}}, x>1.04$, a new peak appears centered near $m / z=19200$ (Figure 2), independent of further increase in $f$ (Figure S7); in Figure 2 we show the behavior for a $33 \%$ (i.e., $1.04 \leq x<1.38$ ) increase in the fluence. The shapes of both peaks (the one at $m / z=24600$ and the other at 19 200) exhibit interesting features (see Figure 2, inset): (i) the former shows a steeply falling edge on the high-mass side, which may be used to estimate the total molecular weight of $\mathbf{1}$, whereas (ii) the latter shows a steeply rising edge on its lowmass side, which may be used to identify a particularly stable inorganic (consisting of silver and sulfur) core. The midpoints of these respective features (fall and rise) occur $\sim 6.3 \mathrm{kDa}$ apart, that is, near $24.8 \mathrm{kDa}$ and $18.5 \mathrm{kDa}$; the organic content of 60 thiolates is also $6.3 \mathrm{kDa}\left(60 \times 105 \mathrm{Da}\right.$, the mass of $\mathrm{PhCH}_{2} \mathrm{CH}_{2}$ 


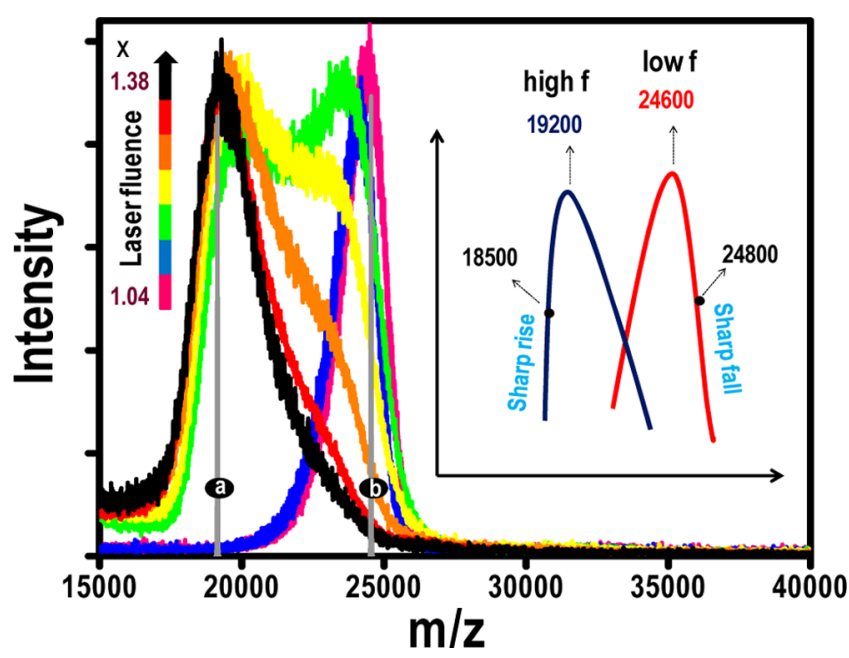

Figure 2. Laser fluence-dependent MALDI mass spectra of the $\mathrm{Ag}_{152}$ compound 1. With a $33 \%$ increase in laser fluence $(1.04<x<1.38)$, the peak position shifts from a higher mass (pink in color with $\mathrm{m} / z$ of 24600 , marked by "b") to a lower mass (black in color with $m / z$ of 19 200, marked by "a"). A further increase in the laser fluence does not change the peak position. Inset: schematic showing the sharp rise and steep fall of the mass-spectral features occurring as the laser fluence is varied.

fragment). These masses and mass-differences suggest that the composition of $\mathbf{1}$ is best described by $\mathrm{Ag}_{152}\left(\mathrm{SCH}_{2} \mathrm{CH}_{2} \mathrm{Ph}\right)_{60}$ for which the inorganic core is $\mathrm{Ag}_{152} \mathrm{~S}_{60}$. This composition is used below as a starting point to obtain a detailed theoretical study to reveal the structure of this cluster.

The toluene extract of $\mathbf{1}$ was dark brown, and it showed an absorption maximum at $460 \mathrm{~nm}(2.69 \mathrm{eV}$; Figure 3). The above band was along with an absorption onset near $1.07 \mathrm{eV}$ (Figure $\mathrm{S} 8$ ), which is comparable with the findings for $\mathrm{Au}_{144}$. Normally

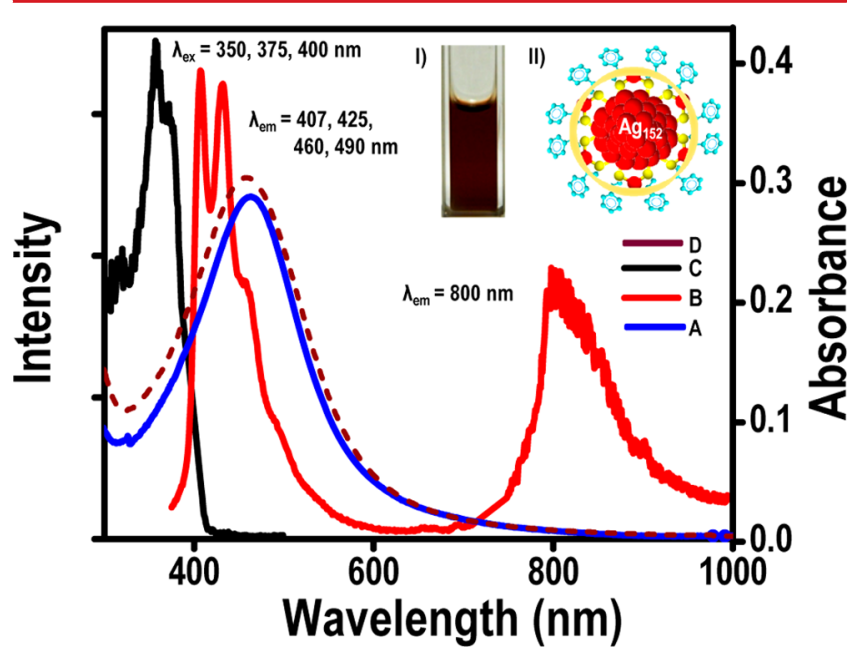

Figure 3. UV-vis absorption spectrum (A) and luminescence spectra ( $\mathrm{B}$ and $\mathrm{C}$ ) of compound 1. The absorption spectrum is reminiscent of $\mathrm{Ag}$ nanoparticles. For comparison, the spectrum of PET protected $\mathrm{Ag}$ nanoparticles of the $5 \mathrm{~nm}$ core diameter is shown (brown dashed line). A NIR emission at $800 \mathrm{~nm}$ was observed when the cluster was excited at $375 \mathrm{~nm}$ (B). The lower wavelength emission may be due to the thiolate shell consisting of the staple motifs. The cluster extracted in toluene is deep brown in color, and a photograph taken in cuvette is shown in inset I. Inset II is a cartoon representation of the cluster. For simplicity, only one type of staple is given in the cartoon.
PET protected clusters do not show any visually observable luminescence under UV light. ${ }^{24}$ However, the as-synthesized cluster emits in the near-infrared (NIR) region with an emission wavelength of $800 \mathrm{~nm}$, upon excitation at $375 \mathrm{~nm}$ (Figure 3). This NIR emission is attributed to the silver core. The fine structure at the lower wavelength region (400-600 $\mathrm{nm}$ ) of the emission spectrum may be due to the protective silver-thiolate network (see theory section below); this assignment is supported by the fact that the features exhibit strong structures reminiscent of metal complexes. The optical absorption spectrum of the $\sim 2 \mathrm{~nm} \mathrm{Ag}$ particles on which we focus here resembles the plasmon excitation of silver nanoparticles of $5 \pm 2 \mathrm{~nm}$ core and above, protected with PET (Figure 3). A TEM image of these nanoparticles is shown in Figure S9. Beyond this core size, the spectrum does not change. It may be noted that the spectral width of the two (compound $\mathbf{1}$ and larger nanoparticles) is also comparable. The HPLC-separated cluster shows an identical optical absorption spectrum (Figure S2, inset).

The construction of the structural model for the $\operatorname{Ag}_{152}(\mathrm{SR})_{60}$ cluster, described above, was guided by combined experiences derived from investigations on clusters [e.g., bare metal clusters ${ }^{1,17,27,28}$ as well as organometallic clusters containing a metal core protected by an organic shell ${ }^{1,3,12,19,22,29}$ ] and from recent surface science studies of self-assembled monolayer (SAM)-induced reconstruction of the surface of silver. ${ }^{30,31}$ We describe the concentric aufbau methodology by starting from the inner region of the silver cluster (see Figure 4) which we set up as a (hollow) 20-Ag atom dodecahedron, which is a Platonic polyhedron ${ }^{32}$ made of 12 pentagonal faces and 20 vertices, with three faces meeting at each vertex (a signature that is denoted as (5.3)). Each of the 12 pentagonal faces is then capped by an $\mathrm{Ag}$ atom (located above the midpoint of the face), with the capping atoms forming a regular icosahedron (Ih), which is the dual of the underlying dodecahedron (for a layer-by-layer illustration, see Figure S10). The 32-atom inner shell is encapsulated by a $60 \mathrm{Ag}$ atom second shell, with all of the atoms being symmetry-equivalent, forming the Archimedean polyhedron known as a snub-dodecahedron; ${ }^{32}$ this polyhedron is made of 80 triangular faces and 12 pentagonal ones, with five faces (four triangles and one pentagon) meeting at each vertex, that is, a $\left(3^{4} .5\right)$ signature. We note here that this 60 -atom shell is enantiomorphic, that is it appears in right- or left-handed forms. The $(32+60)-\mathrm{Ag}$ atom core cluster described above (see Figure 4, center column) is protected by a shell containing 120 sites that are occupied by $60 \mathrm{Ag}$ atoms and $60 \mathrm{RS}$ - thiolates (Figure 4, right column). This network is made of 20 (distorted) hexagonal units (each made of two unequal triangles rotated by $180^{\circ}$ with respect to each other) with the apexes of one triangle occupied by Ag atoms and those of the other one by RS - groups (that is, going around the distorted hexagon, the locations of the metal and organic molecules alternate). The $\mathrm{Ag}$ sites are positioned above edges of the aforementioned $60 \mathrm{Ag}$ atom snub-dodecahedron second shell, and the thiolates are located radially outward from the underlying $\mathrm{Ag}$ atoms of the second core shell; under firstprinciples relaxation of the structure, the sulfur atoms displace outward with respect to the $\mathrm{Ag}$ atoms in the protective network (see dash-box inset in Figure 4). All of these features-the 1:1 (Ag:RS-) stoichiometry of the protective shell, the honeycomb arrangement, and the alternating height variations of the $\mathrm{Ag}$ and $S$ atoms on the puckered hexagonal rings-bear close similarities to the SAMs-induced reconstruction of the 

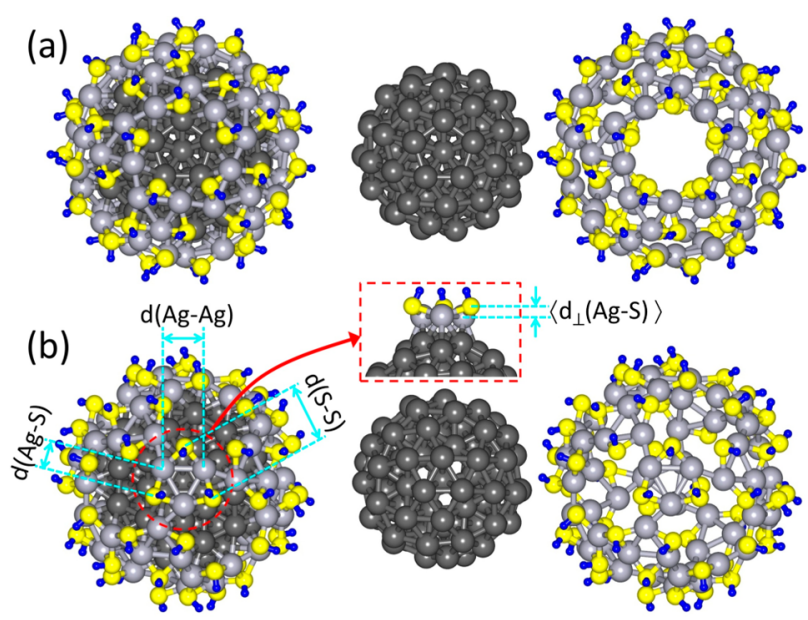

Figure 4. (Left) Optimized structure of the $\mathrm{Ag}_{152} \mathrm{X}_{60}(\mathrm{X}=\mathrm{SH})$ as viewed down a 5-fold symmetry axis (top row, a) and down a 3-fold symmetry axis (bottom row, b). The structure comprises two major substructures: a 92-site core (all $\mathrm{Ag}$ ), shown in the center column and a 120-site protective network displayed in the right column, in which $\mathrm{Ag}$ and $\mathrm{S}$ sites alternate. Across each row, the orientations described above are preserved. The encircled region (dashed in red) in the lower left frame (3-fold axis view) serves to highlight a top-view of the (Ag$\mathrm{SH})_{3}$ (made of a silver, in light gray, and sulfur, in yellow, triangles), with 20 such units covering the entire surface of the structure. The central dashed rectangle (inset) shows a side view of the same sixmember ring unit. In this image, all other six-membered rings have been omitted, for clarity. Pertinent geometrical parameters: radial distances from the center of the $\mathrm{Ag}_{152} \mathrm{X}_{60}$ cluster: $d(20 \mathrm{Ag}$ first shell $)=$ $3.95 \AA ̊$; $d(12 \mathrm{Ag} \mathrm{Ih})=4.72 \AA$; $d(60 \mathrm{Ag}$ second shell $)=6.48 \AA$; $d(60 \mathrm{Ag}$ protective network $)=8.70 \AA \AA ; d(60 \mathrm{Ag}$ protective network $)=9.68 \AA$. . Distances in the inset (central column): $\langle d(\mathrm{Ag}-\mathrm{Ag})\rangle=3.38 \AA$; $\langle d(\mathrm{Ag}-\mathrm{S})\rangle=2.58 \AA ;\langle d(\mathrm{~S}-\mathrm{S})\rangle=4.78 \AA ;\left\langle d_{\perp}(\mathrm{Ag}-\mathrm{S})\right\rangle=0.98 \AA$. Color designation: $S$ atoms are in yellow, $\mathrm{H}$ atoms in blue, internal silver atoms (of the 92 atom metal core) are depicted in dark gray, and silver atoms at exterior sites (the protective network) shown in light gray. The coordinates of the atoms in the optimized $\mathrm{Ag}_{152}(\mathrm{SH})_{60}{ }^{2+}$ structure are given in Figure S14.

$\operatorname{Ag}(111)$ surface. ${ }^{30,31}$ We note here that we found an essentially identical structure for a protected silver cluster comprised of $154 \mathrm{Ag}$ atoms (see Sections S11, S12), with the two added atoms located inside the 20-atom dodecahedron (the inner core noted above); see details in sections Figure S11 and Figure S12. The radius of the protected cluster model that we described above was found to be consistent with that estimated from the SAXS measurements (i.e., $\sim 1.4 \mathrm{~nm}$, that is, larger than the radius of the silver cluster (measured from the center to the edge of the $\mathrm{Ag}-\mathrm{S}$ protecting shell) and smaller than the sum of that radius and the linear extent of the extended thiolate molecule.

Insights into the electronic structure and stability of the protected silver cluster studied here have been gained through calculations using the spin density-functional theory $(\mathrm{SDFT})^{33}$ in conjunction with nonlocal norm-conserving soft pseudopotentials ${ }^{34}$ with the valence $4 \mathrm{~d}^{10}$ and $5 \mathrm{~s}^{1}$ electronic states of the silver atoms, as well as the valence electrons of the sulfur and hydrogen atoms of the protecting network shell, expanded in a plane-wave basis with a 62 Ry kinetic energy cutoff, and employing the Perdew-Burke-Ernzerhof (PBE) functional in the generalized gradient approximation (GGA) to the exchange-correlation corrections. ${ }^{35}$
In Figure 5a,b, we display for the energy-optimized metal core $\mathrm{Ag}_{92}{ }^{2+}$ (see center column in Figure 4) the projected
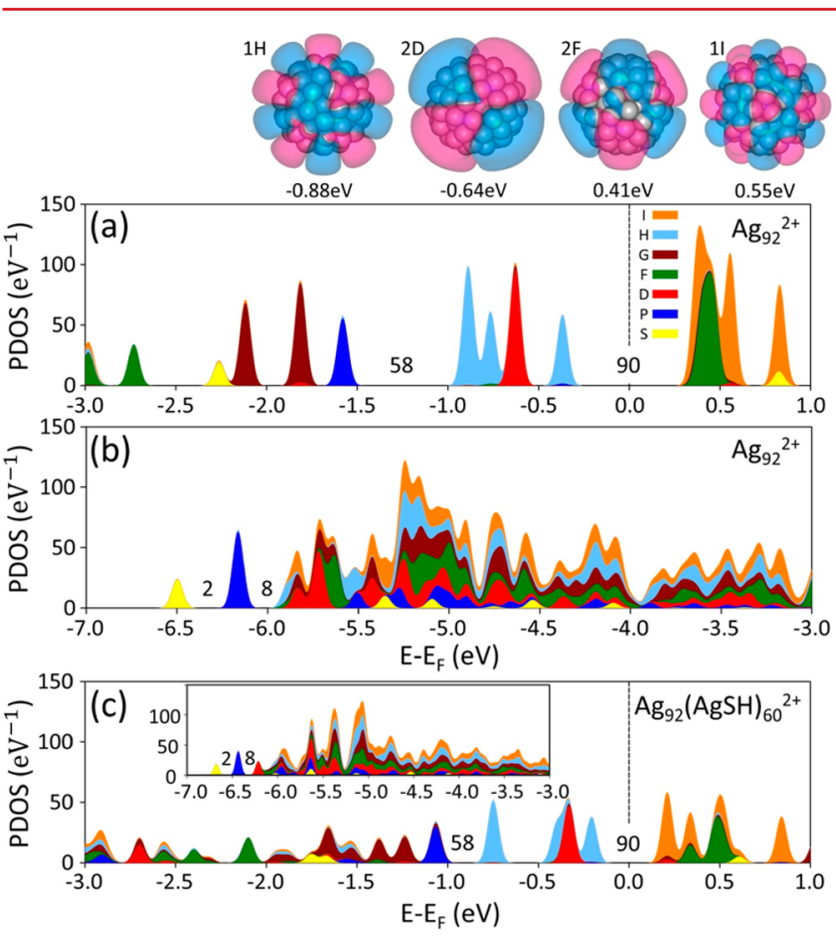

Figure 5. Calculated projected densities of states (PDOS) for $\mathrm{Ag}_{92}{ }^{2+}$ (in a,b) and for $\mathrm{Ag}_{152}(\mathrm{SR})_{60}{ }^{2+}$ (in c, for $\mathrm{R}=\mathrm{H}$ ), obtained through firstprinciples calculations. At the top we show images of selected (delocalized) orbitals near the top of the occupied spectrum, with their energies and angular momenta noted. (blue and pink colors correspond to opposite signs of the wave functions). In the PDOS different colors (see the color code at the right of a) correspond to the various angular momentum contributions, S (0), P (1), D (2), F (3), G (4), H (5), and I (6), which designate the irreducible representations of the full rotation group, $\mathrm{SO}(3)$; the numbers in parentheses denote the angular momenta, $L=0,1,2, \ldots$. , with the number of states being $2 L+1$ for a given $L$ (that is a maximum of $2(2 L+1)$ electrons occupying states of a given angular momentum). The numbers $(2,8,58$, and 90$)$ in the PDOS denote the number of electrons occupying superatom shells corresponding to shell closures. Note also the shell-closure-gap centered near $-2.5 \mathrm{eV}$ (in a) corresponding to 32-electron occupation of the superatom orbitals (with the $2 \mathrm{~S}$ orbital shifted to the top of the gap due to deviation from spherical symmetry). While under perfect spherical, $\mathrm{SO}(3)$, symmetry, the states corresponding to each of the angular momentum components are degenerate, they split in an environment of lower symmetry. Indeed the splitting observed in the calculated PDOS are consistent with the $\mathrm{Ih} / \mathrm{I}$ point group symmetry of the $92 \mathrm{Ag}$ atom core (Figure 4); see in particular the 3:4 weights of the two (split) peaks of the $1 \mathrm{~F}$ level (with the total number of states of F symmetry being 2(3) $+1=7$ ), the $4: 5$ splitting of the states of $1 G$ symmetry, and the 5:3:3 splitting of the states of $1 \mathrm{H}$ symmetry.

density of states (PDOS, i.e. the density of states projected onto the angular momentum components, introduced first in ref 17 , see S13), along with images of representative orbitals near the top of the occupied spectrum; a vertical dashed line at $E-E_{\mathrm{F}}=0$ denotes the location of the midpoint between the highest occupied molecular orbital (HOMO) and the lowest unoccupied one (LUMO). In Figure 5c we show the PDOS for the optimized structure of $\mathrm{Ag}_{92}(\mathrm{AgSH})_{60}{ }^{2+}$ (see Figure 4, left column). The first outstanding feature observed is the large HOMO-LUMO energy gap $\left[\Delta_{\mathrm{HL}}=0.73 \mathrm{eV}(0.41 \mathrm{eV})\right.$ in 
Figure 5a,c]. This large gap confers high stability to the cluster, endowing it with resistance to chemical attack.

Inspection of the electronic structure of the cluster and the orbitals' angular momentum symmetries (Figure 5) shows that, in agreement with an early proposal (introduced first in ref 17 termed as a "partial jellium" model, and used extensively in subsequent studies of protected metal clusters, ${ }^{36}$ while for a wide range of energies (located at the middle of the energy spectrum) the electronic wave functions exhibit localized character (associated with $\mathrm{Ag}$ atomic $4 \mathrm{~d}$ electrons), the orbitals of states with energies near the top and bottom of the electronic spectra are of delocalized character, derived from the atomic $\mathrm{Ag} 5$ s electrons (see representative orbital images in Figure 5a). These delocalized states can be assigned particular symmetries (determined with the use of an expansion of the calculated wave functions in spherical harmonics (see S13) following the electronic cluster-shell-model (CSM), with a (superatom) aufbau rule: $1 \mathrm{~S}^{2}\left|1 \mathrm{P}^{6}\right| 1 \mathrm{D}^{10}\left|2 \mathrm{~S}^{2} 1 \mathrm{~F}^{14}\right| 2 \mathrm{P}^{6} 1 \mathrm{G}^{18} \mid$ $2 \mathrm{D}^{10} 1 \mathrm{H}^{22} 3 \mathrm{~S}^{2} \mid 1 \mathrm{I}^{26}$, and so forth, where $\mathrm{S}, \mathrm{P}, \mathrm{D}, \mathrm{F}, \mathrm{G}, \mathrm{H}$, and $\mathrm{I}$, correspond, respectively, to angular momenta, $L=0,1,2,3,4$, 5 , and 6 . In the above CSM scheme, the vertical lines denote shell-closures (magic numbers), with each closure accompanied by the opening of a stabilizing energy gap; in the above shellstructure scheme the shell closures occur at $n^{*}=2,8,18,34$, 58,92 , and so forth, electrons (with the values of $n^{*}$ 's called "magic numbers"). In Figure 5 the HOMO complex, consisting of 16 orbitals and holding 32 electrons, consisting of $\left(2 \mathrm{D}^{10}\right.$ $1 \mathrm{H}^{22}$ ) and corresponding to an $\mathrm{Ag}_{92}{ }^{2+}$ core; note ionization of the $3 S^{2}$ level yielding the dication. The LUMO complex consists of 21 (empty) orbitals, arising from 1I, 2F, and $3 S$ states.

Deviations from spherical symmetry can cause certain alterations in level ordering, as well as splittings of the $(2 \mathrm{~L}+$ 1)-fold level degeneracy by crystal-field effects. Such splittings are seen clearly in the PDOS for the $\mathrm{Ag}_{92}{ }^{2+}$ core (see in particular Figure 5a), and they are consistent with the $\mathrm{I}_{\mathrm{h}} / \mathrm{I}$ point group symmetry of the core. Furthermore, we note that these splittings are maintained also in the electronic spectrum of the protected $\mathrm{Ag}_{152}(\mathrm{SR})_{60}{ }^{2+}$ cluster (see in particular the 5:3:3 splitting of the $1 \mathrm{H}$ states and the degeneracy of the $2 \mathrm{D}$ states at the top of the spectrum (compare Figure 5 a and c), attesting to the (autonomous) integrity of the protective silver-thiolate network (Figure 4, right column).

In summary, PET protected silver quantum cluster was synthesized using a solid state route. Based on MALDI MS data the cluster composition was assigned to $\mathrm{Ag}_{152}\left(\mathrm{SCH}_{2} \mathrm{CH}_{2} \mathrm{Ph}\right)_{60}$. It shows an optical absorption spectrum reminiscent of silver nanoparticles. It exhibits a NIR emission $\left(\lambda_{\text {emi }} \sim 800 \mathrm{~nm}\right)$ due to the core and structured emission due to the shell. The cluster mass spectrum is sensitive to the laser fluence. Excellent periodic arrangement of the clusters was observed in HRTEM and SEM. Exploiting combined insights from investigations of clusters and surface science, a core-shell structural model was developed, with a 92-atom silver core having icosahedral-snub dodcahedral symmetry and an encapsulating protective shell comprised of $60 \mathrm{Ag}$ atoms and 60 thiolates arranged in a network of six-membered rings resembling the geometry found in self-assembled monolayers on $\mathrm{Ag}(111)$. First-principles electronic structure calculations show the development of a $\sim 0.4$ eV HOMO-LUMO energy gap for the geometryoptimized structure, originating from a superatom 90-electron shell-closure and conferring stability to the cluster when in the $2^{+}$(dication) charge state, in correspondence with the measured large dication intensity in the purified cluster material (see Figure 1b). Efforts are underway to crystallize the clusters.

\section{ASSOCIATED CONTENT}

\section{Supporting Information}

Details of experimental procedures and characterization using UV-vis (absorbance in terms of energy), SEM, EDAX, HPLC, SAXS, and MALDI MS of the $25 \mathrm{kDa}$ clusters at varying laser intensities. For the theory part we report the method for calculations of the PDOS, a layer-by-layer construction of the 92-atom metal core of the cluster, and the coordinates of all the atoms in the optimized structure of the $\mathrm{Ag}_{92}(\mathrm{SH})_{60}{ }^{2+}$ cluster. This material is available free of charge via the Internet at http://pubs.acs.org.

\section{AUTHOR INFORMATION}

\section{Corresponding Author}

*E-mail: pradeep@iitm.ac.in. Fax: + 91-44 2257-0545.

\section{Present Address}

${ }^{\perp}$ Summer students from Stella Maris College, Chennai, India. Author Contributions

"These authors contributed equally.

\section{Notes}

The authors declare no competing financial interest.

\section{ACKNOWLEDGMENTS}

We thank the Department of Science and Technology, Government of India and IIT Madras for constantly supporting our research program on nanomaterials. Thanks to CGCRI, Kolkata for the SAXS analysis. The work of B.Y. and U.L. was supported by the Office of Basic Energy Sciences of the US Department of Energy under Contract No. FG05-86ER45234 and in part by a grant the Air Force Office of Scientific Research. Computations were made at the GATECH Center for Computational Materials Science.

\section{REFERENCES}

(1) Whetten, R. L.; Khoury, J. T.; Alvarez, M. M.; Murthy, S. Vezmar, I.; Wang, Z. L.; Stephens, P. W.; Cleveland, C. L.; Luedtke, W. D.; Landman, U. Adv. Mater. 1996, 8, 428-433.

(2) Zheng, J.; Nicovich, P. R.; Dickson, R. M. Annu. Rev. Phys. Chem. 2007, 58, 409-431.

(3) Jin, R. Nanoscale 2010, 2, 343-362.

(4) Shichibu, Y.; Suzuki, K.; Konishi, K. Nanoscale 2012, 4, 41254129.

(5) Schmid, G.; Meyer-Zaika, W.; Pugin, R; Sawitowski, T.; Majoral, J.-P.; Caminade, A.-M.; Turrin, C.-O. Chem.-Eur. J. 2000, 6, 16931697.

(6) Schmid, G. Chem. Soc. Rev. 2008, 37, 1909-1930.

(7) Shichibu, Y.; Negishi, Y.; Tsukuda, T.; Teranishi, T. J. Am. Chem. Soc. 2005, 127, 13464-13465.

(8) Shichibu, Y.; Negishi, Y.; Tsunoyama, H.; Kanehara, M.; Teranishi, T.; Tsukuda, T. Small 2007, 3, 835-839.

(9) Shibu, E. S.; Muhammed, M. A. H.; Tsukuda, T.; Pradeep, T. J. Phys. Chem. C 2008, 112, 12168-12176.

(10) Zhu, M.; Lanni, E.; Garg, N.; Bier, M. E.; Jin, R. J. Am. Chem. Soc. 2008, 130, 1138-1139.

(11) Heaven, M. W.; Dass, A.; White, P. S.; Holt, K. M.; Murray, R W. J. Am. Chem. Soc. 2008, 130, 3754-3755.

(12) Jadzinsky, P. D.; Calero, G.; Ackerson, C. J.; Bushnell, D. A.; Kornberg, R. D. Science 2007, 318, 430-433.

(13) Pei, Y.; Gao, Y.; Zeng, X. C. J. Am. Chem. Soc. 2008, 130, 78307832. 
(14) Rao, T. U. B.; Nataraju, B.; Pradeep, T. J. Am. Chem. Soc. 2010,

132, 16304-16307.

(15) Udaya Bhaskara Rao, T.; Pradeep, T. Angew. Chem., Int. Ed. 2010, 49, 3925-3929.

(16) Chakraborty, I.; Udayabhaskararao, T.; Pradeep, T. Chem. Commun. 2012, 48, 6788-6790.

(17) Yoon, B.; Koskinen, P.; Huber, B.; Kostko, O.; von Issendorff, B.; Häkkinen, H.; Moseler, M.; Landman, U. ChemPhysChem 2007, 8, 157-161.

(18) Knight, W. D.; Clemenger, K.; de Heer, W. A.; Saunders, W. A.; Chou, M. Y.; Cohen, M. L. Phys. Rev. Lett. 1984, 52, 2141-2143.

(19) Branham, M. R.; Douglas, A. D.; Mills, A. J.; Tracy, J. B.; White, P. S.; Murray, R. W. Langmuir 2006, 22, 11376-11383.

(20) Negishi, Y.; Arai, R.; Niihori, Y.; Tsukuda, T. Chem. Commun. 2011, 47, 5693-5695.

(21) Schaaff, T. G.; Shafigullin, M. N.; Khoury, J. T.; Vezmar, I.; Whetten, R. L. J. Phys. Chem. B 2001, 105, 8785-8796.

(22) Lopez-Acevedo, O.; Akola, J.; Whetten, R. L.; GroÎ̀nbeck, H.; Haİkkinen, H. J. Phys. Chem. C 2009, 113, 5035-5038.

(23) Chaki, N. K.; Negishi, Y.; Tsunoyama, H.; Shichibu, Y.; Tsukuda, T. J. Am. Chem. Soc. 2008, 130, 8608-8610.

(24) Qian, H.; Jin, R. Nano Lett. 2009, 9, 4083-4087.

(25) Dass, A. J. Am. Chem. Soc. 2009, 131, 11666-11667.

(26) Dharmaratne, A. C.; Krick, T.; Dass, A. J. Am. Chem. Soc. 2009, 131, 13604-13605.

(27) Martin, T. P. Phys. Rep. 1996, 273, 199-241.

(28) Cleveland, C. L.; Landman, U.; Schaaff, T. G.; Shafigullin, M. N.; Stephens, P. W.; Whetten, R. L. Phys. Rev. Lett. 1997, 79, 18731876.

(29) Tran, N. T.; Powell, D. R.; Dahl, L. F. Angew. Chem., Int. Ed. 2000, 39, 4121-4125.

(30) Parkinson, G. S.; Hentz, A.; Quinn, P. D.; Window, A. J.; Woodruff, D. P.; Bailey, P.; Noakes, T. C. Q. Surf. Sci. 2007, 601, 5057.

(31) Otálvaro, D.; Veening, T.; Brocks, G. J. Phys. Chem. C 2012, 116, 7826-7837.

(32) Williams, R. The Geometrical Foundation of Natural Structure; Dover: New York, 1979.

(33) Barnett, R. N.; Landman, U. Phys. Rev. B 1993, 48, 2081-2097.

(34) Troullier, N.; Martins, J. L. Phys. Rev. B 1991, 43, 1993-2006.

(35) Perdew, J. P.; Burke, K.; Ernzerhof, M. Phys. Rev. Lett. 1997, 78, $1396-1396$.

(36) Walter, M.; Akola, J.; Lopez-Acevedo, O.; Jadzinsky, P. D.; Calero, G.; Ackerson, C. J.; Whetten, R. L.; Groenbeck, H.; Hakkinen, H. Proc. Natl. Acad. Sci. 2008, 105, 9157-9162. 